\title{
Collaborative Algorithm for the Spectrum Allocation in Distributed Cognitive Networks
}

\author{
Camila Salgado ${ }^{1}$, Sergio Mora ${ }^{2}$, Diego Giral ${ }^{3}$ \\ ${ }^{1}$ Associate professor, Department of Electronic Engineering, Universidad ECCI \\ Cr. 19 \#49-20, Bogotá, Colombia \\ lsalgadof@ecci.edu.co \\ ${ }^{2}$ Associate professor, Research Department, Universidad ECCI \\ Cl. 51 \#19-12, Bogotá, Colombia \\ smoram@ecci.edu.co \\ ${ }^{3}$ Professor, Universidad Distrital Franciso José de Caldas \\ Carrera 7 No. 40B - 53, Bogotá, Colombia \\ dagiralr@udistrital.edu.co
}

\begin{abstract}
The objective of this paper aims to benchmark the performance of a proposed collaborative algorithm for the dynamic spectrum allocation in distributed cognitive wireless networks. An algorithm called Collaborative FAHP was developed, it is intended to share the cognitive user's information and from that information select the best spectrum opportunity. The assessment was carried out through the development of simulation software based on the real spectral occupancy data taken from the $1850 \mathrm{MHz}$ to $2000 \mathrm{MHz}$ frequency band. The results were compared with two more algorithms, simple FAHP and completely Random one. The results show that as there is more spectrum information the FAHPCollaborative algorithm increases its performance level regarding bandwidth. However, it is also noted the results are dependent on the data quantity and quality that is shared among the cognitive users.
\end{abstract}

Keywords-Collaborative algorithm, machine learning, dynamic spectrum access, artificial intelligence, cognitive radio, distributed networks.

\section{INTRODUCTION}

The rapid grow of the new mobile services, the anticipated scarcity of the radioelectric spectrum and the vast underutilization of it, have motivated the use of the Dynamic Spectrum Access (DSA) model [1] and is based on the set of supported techniques in the information theories and telecommunications. Thus, the DSA concept comprises action areas as the principles of optimization, artificial intelligence, automatic learning, among others [2].

In the cognitive radio (CR), DSA increases the spectral efficiency of the system, allowing the unlicensed users or cognitive users $(\mathrm{CU})$, can use the underused space-time spectrum bands under the precedent of being transparent to the licensed users or primary users (PU) [2]. The availability of a transmission channel is defined as the probability a licensed channel is available and it can be used by a CU. This availability is a critical parameter for the practical design of selection strategies of communication channels [3], [4], [5].

According to the current researches, the dynamic and intelligent allocation of the spectrum in wireless networks, is the essential characteristic to reduce delay, the interference and the number of channel changes (spectrum handoff), during the unlicensed user's transmission or cognitivive user's transmission, because dynamic allocation allows a more efficient use of the spectrum, since not always the licensed user uses during the whole time the spectral and intelligent resource because it will allow selecting the frequency channels with the best characteristics given the service class that is required (real-time or best-effort), such as the occupancy probability of a channel, estimated time of the occupancy channel, signal to noise ratio plus channel interference and channel bandwidth; the aforementioned contributes to minimize the channel degradation as the intelligent algorithm of dynamic spectrum allocation allows reducing the interference caused by the CU to the licensed user. Moreover, during the development of a spectrum handoff strategy, it is needed to count on a frequency channel that allows maximizing the average transmission time, improving the level of the data rate of the CU communication, which is possible if the intelligent algorithm of dynamic allocation selects the frequency channel with the lowest occupancy probability, the lowest average occupancy time by the licensed user, the best signal to noise ratio and the best available bandwidth. 
This research paper presents a solution for the aforementioned problem "An intelligent algorithm that carries out the dynamic spectrum allocation in wireless distributed cognitive networks, improving the spectrum mobility performance of the cognitive users, from the selection criteria: channel occupancy probability, channel occupancy average time, signal to noise ratio, channel bandwidth available, type of traffic and the previous data information." This intelligent algorithm is developed from artificial intelligence techniques. First, there must not be forgotten that the more information is known, that is to say, the more explanatory variables are evaluated, better forecasts will carry out, and consequently the proposed model must be multicriteria. For instance, having information of the available resources in the cognitive radio network will determine with better precision the need of carrying out a spectrum handoff.

In accordance with the aforestated, the objective of the current research aims to design, develop and evaluate an intelligent algorithm for the dynamic spectrum allocation in wireless distributed cognitive networks, improving the spectrum mobility performance of the cognitive users, from the selection criteria: channel occupancy probability, channel occupancy average time, signal to noise ratio, channel bandwidth available and evaluation metrics: average number of necessary and unnecessary channel changes, average bandwidth available, transmission average delay, average transmission data rate, based on obtained real spectrum occupancy data.

The article is structured as follows: in Section 2 a description of related work in current literature is carried out. In Section 3 the research methodology is described. In Section 4 the achieved results in the research are shown. And finally, in Section 5 conclusions are drawn.

\section{RELATED WORK}

In [6] the efficient channel distribution for CRN must have studies and analysis which are the favorable techniques to optimize the spectrum allocation. For this reason, the current paper elaborates a solution through the pondered weights of each channel in function of the system user's activity. For this, it has been considered an ad-hoc network in order to provide a specific solution based on study entrances. Thus, the proposed network has components and characteristics that are based on the PU node set and the CU node configuration, hence the main assumptions arise from the need to establish Multihop Wireless Networks as efficient methodology that enables to provide greater coverage due to the multi-hop and moreover improving the performance in channel jumps in the current coverage in the communication links.

On that basis, the research establishes the creation of a CRN with the ability of classifying channels in function of their weights. Moreover, the classification is in function of the no-occupancy of PU users and the number of $\mathrm{CU}$ neighbors, employing the performance of each channel to achieve greater connectivity finally through the selection of spectrum bands and assuring decrease in the transmitted data error rate. Lastly, it is worth highlighting the proposed model has had good results in comparison with the traditional methods based on random selection (Random Channel selections) and selective diffusion (Selective Broadcast).

In [7] there is an optimization algorithm in order to improve the decision-making process in the use of radio resources in heterogeneous wireless cognitive networks. In the networks with centralized management, a new optimization algorithm is proposed whose solution is assured to be exactly optimum. To avoid an exponential increase in the computational complexity in wireless networks in large scale, the optimization problem is shaped as a problem of minimum cost flow and a solution is found for the problem in polynomial time. For the networks with decentralized management, a distributed algorithm is proposed, employing the minimization dynamic of the distributed energy of the Hopfield neuronal network.

In [8] a new approach of the spectrum allocation for distributed CRN is described, taking into consideration that the CR systems are capable of detecting the environmental prevailing conditions and automatically the function parameters adaptation with the aim to improve the system and the network performance. Akin into account the capacities of $\mathrm{CR}$ the approach of this research is focused on optimizing each individual wireless device and its communication links with only one hop using the information of the environment of the near device within the wireless network. Assuming wireless stationary networks, all the wireless communication links employ Non Contiguous Orthogonal Frequency division multiplexing (NC-OFDM) with the goal of allowing the dynamic spectrum access (DSA). The proposed approach tries to reduce to the minimum the bit error rate, minimizing the out-of-band $(\mathrm{OOB})$ interference and maximizing the general performance using a function of multi-objective adequacy. For this, genetic algorithms are employed which are used to develop the necessary optimization.

In [9] a study about resource allocation in cooperative CRN is presented. The objectives and protocols used in the literature for the resource allocation in cooperative CRN were also discussed. It stands out the use of power control, types of cooperation, the network configuration and types of used decision in cooperative CRN. The systems of cooperative communication can increase the data velocity of the wireless communication systems with the same power values and bandwidth in wireless communication systems. If CR takes into consideration the cooperative communication concept, this could mean a greater performance in the wireless network.

In [10] the performance of the channel selection algorithm, SURF and Selective Diffusion (SB) is analyzed in order to select distributed channel in CRN. CR is able to identify the bands with a license that are being 
underused in the spectrum and allows the re-use to the $\mathrm{CU}$, each time that the channel is empty. The selection method of the distributed channel is used for the transference of data in multiple hops. From there, the average relation of delivery is improved and the harmful interferences caused by the primary radio nodes are reduced considerably. Through the simulations in NS-2, the results are analyzed, and it is confirmed that SURF is the most efficient method for the channel selection.

In [11] it is proposed a strategy of selection of intelligent channel and distributed for the efficient data diffusion in multiple jumps in the CR network. The strategy, SURF, classifies the available channels and use them in an efficient way to increase the data diffusion reliability in the multi-hop CRN. The classification is carried out based on the no-occupancy by the primary user and the number of neighbors of CR that are using the channels. Through extensive simulations in NS-2, the behavior of SURF is studied in comparison with four related approaches. The simulation results confirm SURF is efficient in the selection of the best channels for an efficient communication and maximum diffusion accessibility in multi-hop CRN.

In document [12] the design of a protocol is made, which is distributed for Medium Access Control (MAC), assisting the channel access of the CU. The proposed MAC protocol, allows an access free of collisions, for the available data channels; and, its use by CU. It is highlighted the part of spectrum detection, which is applied by the nodes, as exclusive sensors. It is also implemented, the provision of the reserve of free channels, by CU during prolong periods; in order to increase its use without causing harmful interferences in PU. The authors [12], show how the wide access to resources, it can be adjusted to provide a differential quality to the service of CU.

Finally, the protocol is evaluated through a simulation. In optimum conditions, the proposed system allows the $\mathrm{CU}$ in the network, using all the available channels, overcoming all the existent MAC schemes in the distributed networks.

\section{METHODOLOGY}

The methodology of the current research paper is structured in five main phases: (1) equipment and software used; (2) the capture and processing of the real spectral data (3) the design of the FAHP-Collaborative algorithm (4) the evaluation and validation of the FAHP-Collaborative algorithm; and (5) simulation software design.

\section{A. Equipment and Software}

For the first and third phase of the current research project, it was only necessary to count with a computer equipment (TABLE I) capable of carrying out the necessary information processing to achieve a good agreement in the development and the evaluation of FAHP-Collaborative algorithm, thus as two licensed software used: Matlab and Excel. For the development of the second phase of the research project it was necessary to link specialized equipment (TABLE II).

TABLE I.

Technical specifications of computer equipment

\begin{tabular}{|l|l|}
\hline \multicolumn{1}{|c|}{ Characteristic } & \multicolumn{1}{c|}{ Description } \\
\hline Equipment and Brand & Asus Laptop \\
\hline Serial & N551JK \\
\hline Processor & Core i7 \\
\hline RAM & 8GB (DDR3) \\
\hline Hard Drive & 700GB SSD \\
\hline Operative System & Windows 8, 64 bits \\
\hline
\end{tabular}

Source: Elaborated by the author

TABLE II.

Technical specifications of the spectral measurement equipment

\begin{tabular}{|l|l|l|}
\hline \multirow{2}{*}{\multicolumn{1}{|c|}{ Equipment }} & \multicolumn{2}{c|}{ Specifications } \\
\cline { 2 - 3 } & \multicolumn{1}{c|}{ Frequency Range } & \multicolumn{1}{c|}{ Reference } \\
\hline Discone antenna type & $25 \mathrm{MHz}-6 \mathrm{GHz}$ & Super-M Ultra Base \\
\hline Broadband cable & $\mathrm{DC}-18 \mathrm{GHz}$ & CBL-6FT SMNM+ \\
\hline Low noise amplifiers & $20 \mathrm{MHz}-8 \mathrm{GHz}$ & ZX60-8008E-S + \\
\hline Spectrum analyzer & $9 \mathrm{KHz}-7.1 \mathrm{GHz}$ & MS2721B Anritsu \\
\hline
\end{tabular}

Source: Elaborated by the author 


\section{B. Capture and Processing of Spectral Data}

The methodology for the data captured of spectrum occupancy is based on the needs and the requirements of the technic evaluation and validation of the developed FAHP-Collaborative algorithm. Firstly, determining the wireless networks application that was desired to work was conducted, for which the cellular communications were selected, because of their high demand and low quality service level, hoping to be able to carry out a meaningful input. Secondly, the cellular technology was selected, for which the band $1850 \mathrm{MHz}$ to $2000 \mathrm{MHz}$ was selected. Thirdly, the spectrum detection technique was selected, for which the energy detection technique was chosen, due to its easy implementation and low requirements. The technical specifications of the equipment and devices are shown in TABLE II. Fourth, the frequency channels were selected corresponding to the frequency band between $1850 \mathrm{MHz}$ to $2000 \mathrm{MHz}$. Fifth, a capture time of a month was determined, from which an hour was selected in order to carry out the corresponding evaluations and validations of the developed algorithm. Lastly, the technical parameters of the spectrum analyzer were configured and it was proceed to capture the measurements.

The band measuring ranges were based on aspects as the sweep time that oscillated about 333 milliseconds, the resolution bandwidth (RBW), which was about $3 \mathrm{KHz} \leq \mathrm{RBW} \leq 100 \mathrm{KHz}$ and the span with a value of $50 \mathrm{MHz}$, with the purpose of assuring a proper measure in function of the floor noise and the channel bandwidth of the technology to be measured [13].

To determine if the signals are present or not, the signal power level was compared to a decision threshold of $5 \mathrm{dbm}$ over the average floor noise level of the frequency band if the value of the signal is higher than the decision threshold it is concluded the channel is occupied otherwise the channel is available.

Once the spectrum occupancy data were captured, the processing of proceedings was carried out, with the aim of calculating the values of each one of the subcriteria which were determined in the hierarchy, and unify the different traces of spectrum occupancy delivered by the spectrum analyzer, conforming a complete database that carries out the required calculations by the evaluation process of the developed Collaborative-FAHP algorithm.

An important aspect in this data processing, is the fact that once calculated the estimated values of each one of the selected subcriteria, it proceeded to carry out the normalization of the aforementioned, in order that a high value in one of the subcriteria with less percentage does not mask a low value in one of the subcriteria with greater importance percentage.

The value of each one of the subcriteria corresponds to the estimated value of the captured data sample; it was decided not to work with the value in real time due to the computational cost of such calculations. Only, it was employed in real time the variable that describes if the channel is occupied or available.

The decision subcriteria by which each one of the spectrum opportunities are selected, were carefully chosen and correspond to the followings:

- Probability of channel availability

- Estimated time of channel availability

- Signal to noise ratio of the cannel (SINR)

- Channel bandwidth

\section{FAHP Collaborative Algorithm Design}

Down below the development of the FAHP collaborative algorithm is described, it is based on the FAHP algorithm. Initially, the FAHP algorithm is developed then; the next five steps are followed:

1) First step statement of the problem: In the design methodology of the FAHP algorithm, the first step is stating the problem and also, objectives, criteria (sub-criteria if there are) and alternatives.

In this paper, the objective of the decision to be taken is significantly clear and corresponds to the selection of the best option of the available backup channel for cognitive radio networks.

The criteria and sub-criteria are the factors that affect the preferences of an alternative, due to the importance that the election of these parameters has several expert people were consulted by means of two inquiries implemented in two different time phases on the issue about spectrum mobility in cognitive radio. The first survey aimed to define which criteria and sub-criteria should be taken into consideration to establish the FAHP algorithm hierarchy and the second one aimed to detect the corresponding weights to each criterion and subcriterion of that hierarchy. The procedure that was made to calculate the criteria and sub-criteria is an approximation of Delphi method.

The alternatives are all the existing options, and only one has to be chosen, in this case, they correspond to the frequency channels that are available when the Collaborative FAHP algorithm is executed. 
2) Second step construction of the hierarchy: The selected criteria and sub-criteria, together with the objective and the alternatives of the FAHP, the hierarchy was constructed.

3) Third step judgment matrices: Once the hierarchy was constructed, the judgment matrices were done, they correspond to the benchmarking that defines the relative importance level among each possible combination of criteria and sub-criteria couple independently. Considering that benchmarking demands an extensive and precise knowledge about the topic, a second survey was implemented on the selected experts.

According to Büyüközkan "people in charge of decision making usually find to feel better by presenting their judgments as an interval, instead of providing a punctual and fixed value. This is because he, she or they, are incapable of explaining their preferences, due to the diffuse nature on the human comparison processes" [14], Thus, it was decided to work with a triangular fuzzy numbers scale presented in TABLE III, from which it takes the connotation of AHP fuzzy algorithm to obtain the FAHP method. The fuzzy verbal scale was derived from the conversion of the nine level fuzzy numbers verbal scale presented by Büyüközkan in [14], which has been extensively used because of this decision-making method in several types of research.

TABLE III.

Triangular fuzzy numbers verbal scale

\begin{tabular}{|l|l|l|l|}
\hline \multicolumn{1}{|c|}{ Nomenclature } & \multicolumn{1}{|c|}{ Verbal Scale } & $\begin{array}{l}\text { Triangular Fuzzy } \\
\text { Scale }\end{array}$ & $\begin{array}{l}\text { Triangular Reciprocal } \\
\text { Fuzzy Scale }\end{array}$ \\
\hline AMEI & Absolutely Less Important & $(2 / 7,1 / 3,2 / 5)$ & $(5 / 2,3,7 / 2)$ \\
\hline MFMEI & Very Strongly Less Important & $(1 / 3,2 / 5,1 / 2)$ & $(2,5 / 2,3)$ \\
\hline FMEI & Strongly Less Important & $(2 / 5,1 / 2,2 / 3)$ & $(3 / 2,2,5 / 2)$ \\
\hline DMEI & Weakly Less Important & $(1 / 2,2 / 3,1)$ & $(1,3 / 2,2)$ \\
\hline II & Equally Important & $(1 / 2,1,3 / 2)$ & $(2 / 3,1,2)$ \\
\hline DMAI & Weakly More Important & $(1,3 / 2,2)$ & $(1 / 2,2 / 3,1)$ \\
\hline FMAI & Strongly More Important & $(3 / 2,2,5 / 2)$ & $(2 / 5,1 / 2,2 / 3)$ \\
\hline MFMAI & Very Strongly More Important & $(2,5 / 2,3)$ & $(1 / 3,2 / 5,1 / 2)$ \\
\hline AMAI & Absolutely More Important & $(5 / 2,3,7 / 2)$ & $(2 / 7,1 / 3,2 / 5)$ \\
\hline
\end{tabular}

Source: taken from [14]

Based on the fuzzy verbal scale graphed in TABLE III, the experts delimited the relative importance level of each pair of criteria and sub-criteria respectively through judgment matrices paths, following the instructions presented in the second survey.

4) Fourth step calculation of standardized weight: With the judgment matrices already delimited, the next step was to calculate the standardized weights for each sub-criterion, based on the model suggested by Eduardo Miranda in [15] due to its simplicity and quality of results, based on the geometric mean.

The first step is to calculate the geometric mean $V_{i}$ of each line of the judgment matrix, defined for a specific criterion, which is defined by Equation (1).

$$
v_{i}=\sqrt[n]{\prod_{j=1}^{n} a_{i j}}
$$

Where:

$V_{i}$ is the geometric mean of line $\mathrm{i}$,

$n$ is the number of sub-criteria,

$j$ stands for the columns of the judgment matrix y

$a_{i j}$ is the value of the element of line $\mathrm{i}$ and column $\mathrm{j}$.

The second step is to calculate the vector of eigenvalues $r$, which defines the standardizer weights of each subcriterion, which is defined by Equation (2). 


$$
r=\left[r_{1}, r_{2}, \ldots, r_{n}\right] \text { with } r_{i}=\frac{v_{i}}{\sum_{j=1}^{n} v_{j}}
$$

Where:

$r$ is the vector of eigenvalues,

$r_{1}, r_{2}, \ldots, r_{n}$ is the value of the weights of each sub-criterion,

$v_{i}$ is the geometric mean of line $\mathrm{i}$, and

$v_{j}$ is the geometric mean of column $\mathrm{j}$.

5) Fifth step calculation of Attenberg limits: With the aim of evaluating the validation of the developed FAHP algorithm the consistency rate was calculated, if the consistency rate is lower than 0.1 , the development of the algorithm is satisfactory. This rate indicates how far the perfect consistency estimation is. To calculate the consistency rate, the model suggested by Eduardo Miranda was implemented [15], which is defined by Equation (3).

$$
C I=\frac{\sqrt{\sum_{i=1}^{n} \sum_{j>1}^{n}\left(\ln a_{i j}-\ln \frac{v_{i}}{v_{j}}\right)^{2}}}{\frac{(n-1) \times(n-2)}{2}}
$$

Where:

$C I$ is the consistency rate

$n$ is the number of sub-criteria

$i$ stands for the value of the line of the judgment matrix,

$j$ is the value of the column of the judgment matrix

$a_{i j}$ is the element of line $\mathrm{i}$ and column $\mathrm{j}$

$v_{i}$ is the geometric mean of line $\mathrm{i}$ and,

$v_{j}$ stands for the geometric mean of column $\mathrm{j}$.

6) Collaborative component: The collaborative component of FAHP algorithm is carried out by interchanging information with the rest of the CU that are situated in the same geographic area of the CU that wants to do its transmission. However, it does not have to be transmitting in order not to reduce its data transmission rate. So as to the data transmission rate does as little as possible regarding communication system resources, each CU stores the spectrum opportunities that have been analyzed in the most recent time period in a vector. In that vector, every spectrum opportunity identification is stored, its corresponding average values of the four selected decision criteria: availability probability, estimated time of availability, SINR and bandwidth, during the most recent detection and analysis period as well as length of that period because the aforementioned will average that information with the information shared by the rest of the $\mathrm{CU}$ and the one that the $\mathrm{CU}$ has and wants to transmit.

The higher the number of $\mathrm{CU}$ that share information, the more information gathered about the characteristics of the spectrum opportunities, thus, the better the selection of the requires spectrum opportunity, improving the performance level, of each one of the evaluation metrics of the CU communication. Hence, the number of CU is an essential parameter in the developed simulation software.

\section{Collaborative FAHP Algorithm Evaluation and Validation}

The collaborative FAHP algorithm evaluation was implemented based on the comparative analysis of the performance of the developed algorithms with the no collaborative FAHP and totally random algorithms. The comparisons of the performance level were carried out based on three evaluation metrics: number of made handoff, number of failed handoff and used bandwidth. So as to obtain the corresponding values of each evaluation metric, simulation software that was designed and developed in this research project was used, it depicts the following section. Moreover, to carry out a further analysis of the behavior of each algorithm, two types of PU traffic were taken into consideration, the first one, and real spectrum occupancy data trace where PU covers the frequency channels in a high percentage, reducing considerably the spectrum opportunities and the second one in which there is a greater number of spectrum opportunities.

Because of the reasons aforementioned in the Collaborative FAHP algorithm design, the number of CU that share information in the Collaborative FAHP algorithm has an important role to evaluate the behavior of that algorithm and analyze the percentage that improves the evaluation metrics when CU cooperate with each other. 


\section{E. Simulation Software Design}

The simulator for the dynamic spectrum allocation in cognitive distributed wireless networks is software that evaluates and validates the performance of an intelligent collaborative algorithm for the spectrum allocation in cognitive wireless networks, based on the real spectrum occupancy data gathered in the $1850 \mathrm{MHz}$ to $2000 \mathrm{MHz}$ band.

The main window of the software contains four sections:

- Project Information

- Quantity of Pus and Channels for Pus

- Handoff Time and Models

- Parameters and Weights

For a proper functioning, the software parameterization has to be implemented according to the number scheme of each section. As follows, each section and proper procedure so as to parameterize in an appropriate manner the simulator are described in detail.

1) Project information: The project information section defines the name and path of the folder where the simulation results will be stored. In the name of the project window, the name of the project will be specified; it is recommendable to type without any gaps, use symbols or use short names. In the Save window, the path where the results of simulation will be stored is indicated. It is advisable not to use too long paths and use symbols.

By default, the simulator creates an "ECCI Cognitive Radio" folder in the defined path in Save in, inside that folder, sub-folders will be set up corresponding to the defined projects.

2) Quantity of PUs and channels for PUs: The Quantity of PU and Channels for PU delimits the traffic level and quantity of channels for PU. The section comprehends two adjustment parameters, the quantity of PUs that define the traffic level between high and low and, the quantity of channels for each user; the maximum value corresponds to 50 channels for a 10 minutes transmission time or 500 for a one minute transmission time.

3) Handoff Time and Models: In Handoff Time and Models section depicts the transmission time of CU and the Handoff algorithm. The simulator has an adjustment in the transmission time of CU from 1 to ten minutes. Four handoff models can be used:

- RANDOM

- FAHP RT

- FAHP BE

- COLLABORATIVE FAHP

4) Parameters and decision criteria: In Parameters and Weights section defines the bandwidth, time range, adjacent channels and weights for the decision criteria. In TABLE IV the simulator parameters are presented and in TABLE V the decision criteria for which the weights of interest can be selected are shown.

TABLE IV

Parameters

\begin{tabular}{|l|l|}
\hline Bandwidth & Fixed bandwidth \\
\hline Time range & $\begin{array}{l}\text { Time period in which the initial value of the decision } \\
\text { criteria are calculated }\end{array}$ \\
\hline Adjacent channels & $\begin{array}{l}\text { Number of adjacent channels to implement a multi- } \\
\text { channel transmission }\end{array}$ \\
\hline
\end{tabular}

Source: Elaborated by the author

TABLE V

Weights

\begin{tabular}{|l|}
\hline Availability probability \\
\hline Average availability time \\
\hline PSINR \\
\hline Average bandwidth \\
\hline
\end{tabular}

Source: Elaborated by the author 
If the user selects the default parameters option and default weights, the simulator establishes default values for the parameters and weights respectively.

5) Simulation: The TABLE VI presents the description of three buttons of the software simulation section.

TABLE VI

Simulation buttons

\begin{tabular}{|l|l|}
\hline Simulate & $\begin{array}{l}\text { If all the sections are established correctly, this button executes the algorithm for the } \\
\text { dynamic spectrum allocation in distributed wireless networks }\end{array}$ \\
\hline New simulation & Erase all the adjusted parameters \\
\hline About & Presents a brief description of the simulator \\
\hline
\end{tabular}

Source: Elaborated by the author

\section{RESULTS}

Down below, the achieved results in the research project are presented, which are organized into three sections: results for bandwidth, results for the number of handoffs and comparative analysis.

\section{A. Results for Bandwidth}

In this segment, the obtained results for bandwidth (BW) are displayed in the Figs 1, 2 and 3. They were obtained through the designed software "simulator for spectrum allocation in cognitive wireless networks". It is perceived that the proposed Collaborative FAHP algorithm grants a higher BW than the selected algorithms in HT as the number of known spectrum opportunities increases.

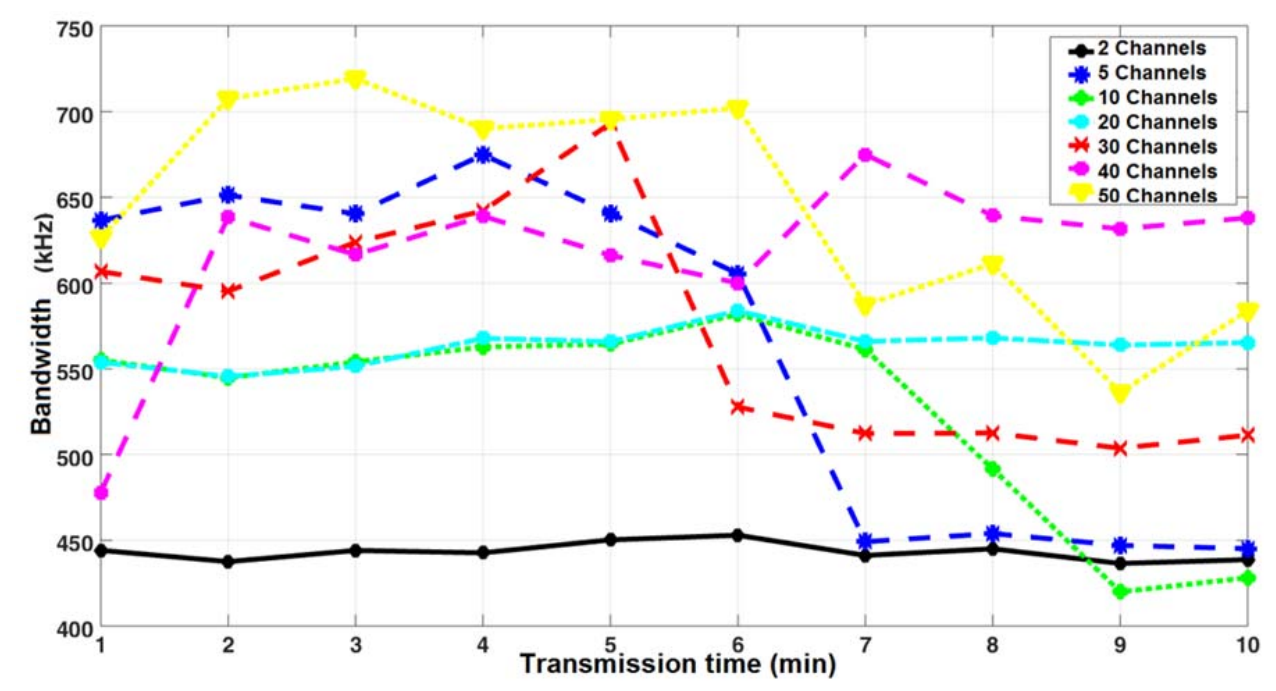

Fig. 1. BW of Collaborative FAHP for different number of channels in HT 


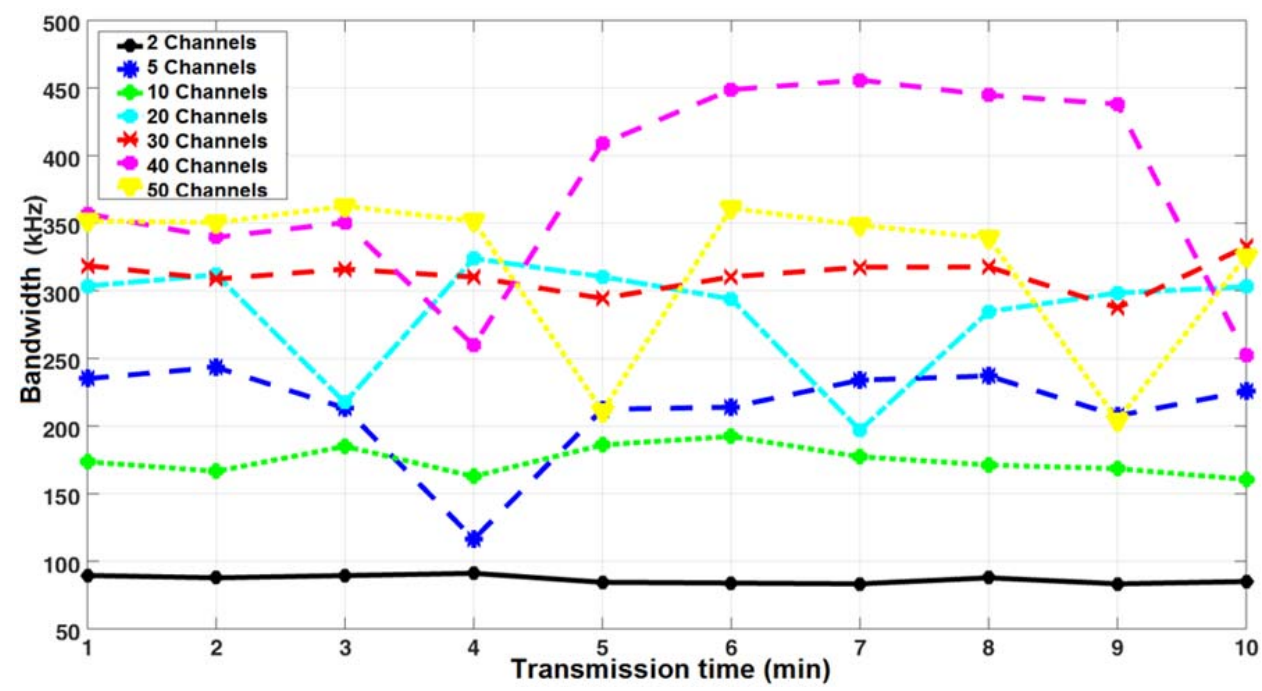

Fig. 2. BW of FAHP for various numbers of channels in HT

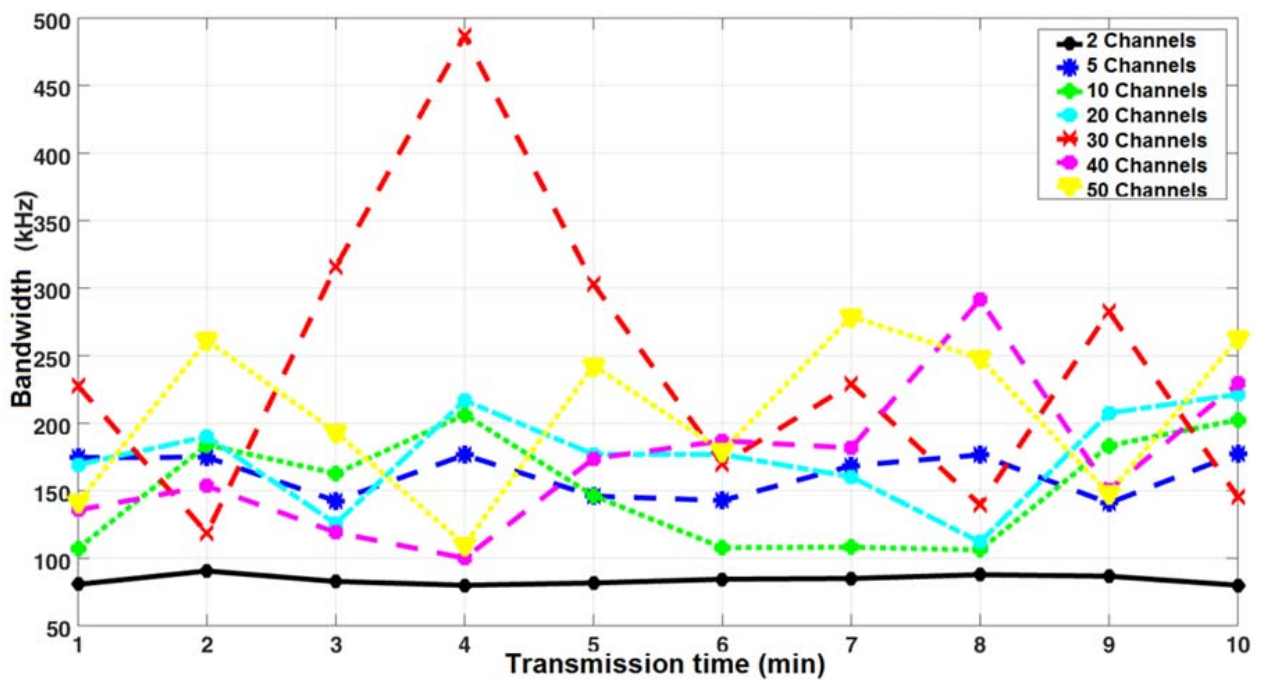

Fig. 3. BW of random algorithm for different number of channels in HT

\section{B. Results for Handoffs}

In this section, the obtained results for Number of Handoff $(\mathrm{H})$ are presented in Figs. 4, 5 and 6; they were gathered using the designed software "simulator for spectrum allocation in cognitive wireless networks." It is observed that Collaborative FAHP algorithm does the lowest quantity of handoff when there is HT in the selected algorithms. 


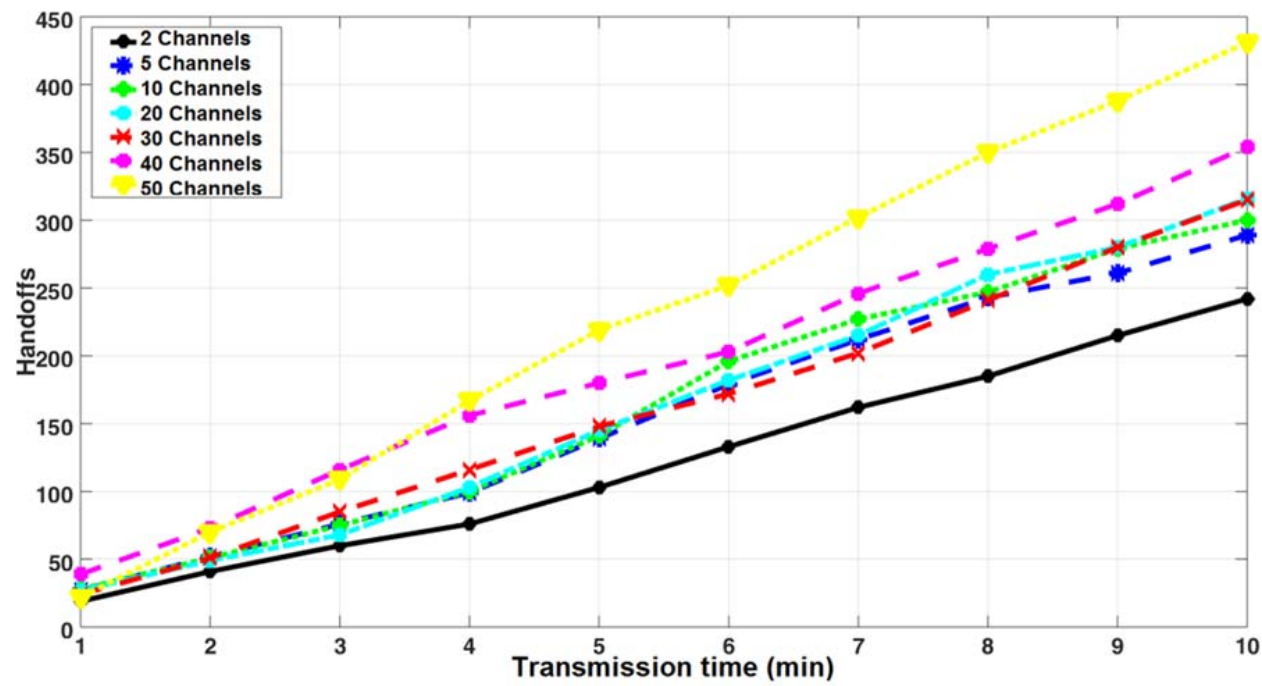

Fig. 4. $\mathrm{H}$ of Collaborative FAHP for different number of channels in HT

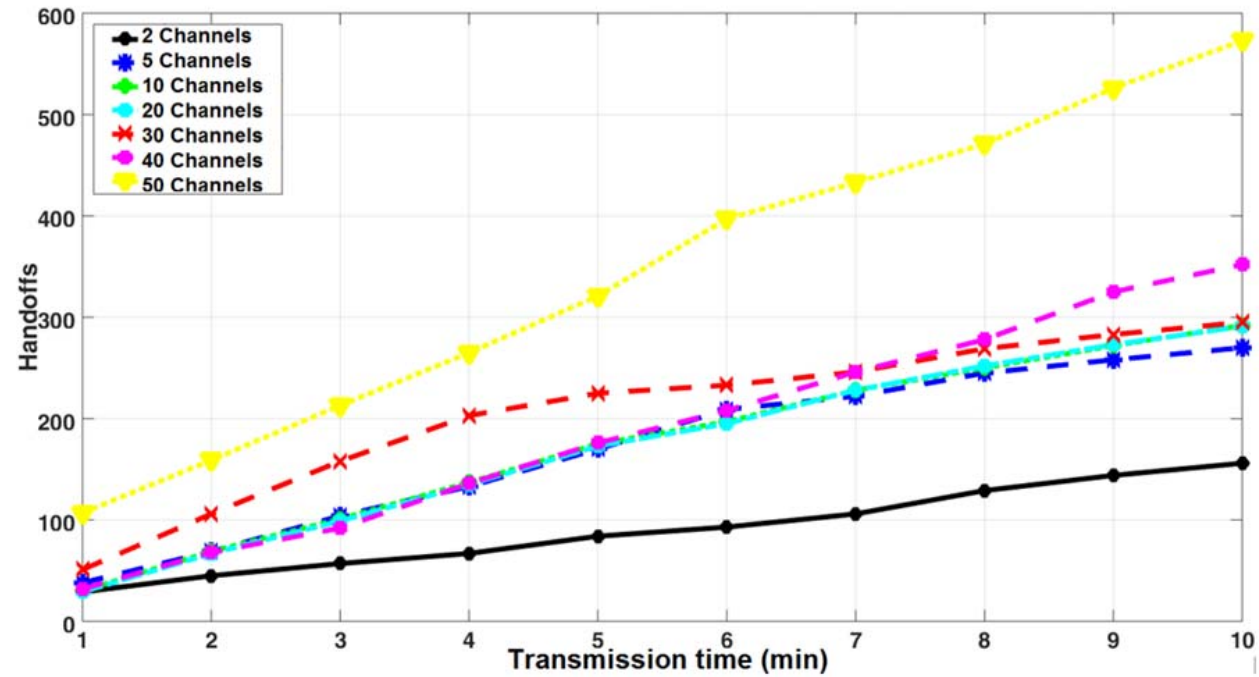

Fig. 5. H of FAHP for different number of channels in HT

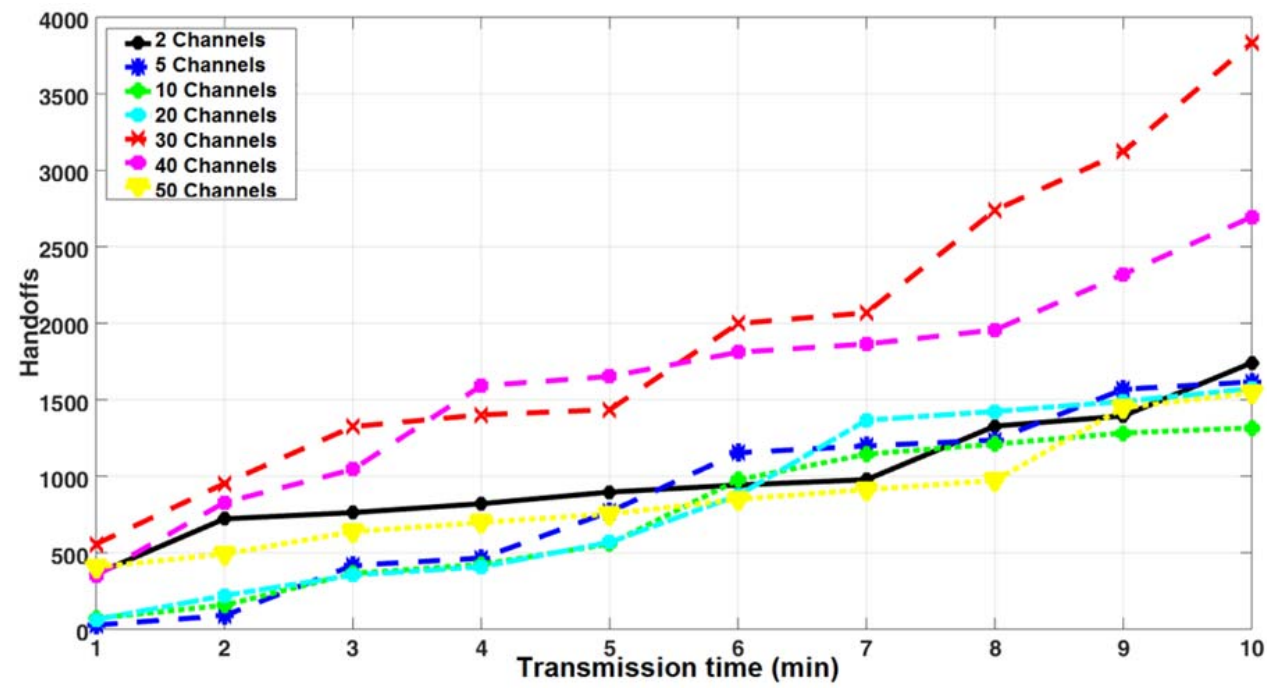

Fig. 6. $\mathrm{H}$ of random algorithm for different number of channels in HT 


\section{Comparative Analysis}

In TABLE VII the performance level of each algorithm for different numbers of channels used during simulations is described. In TABLE VII it is perceived that the random algorithm presents the worst performance, and the proposed Collaborative FAHP algorithm has the best one, with a 7.4\% mark-up on FAHP in the worst scenario and $34.33 \%$ in the best scenario.

TABLE VII

Global benchmarking per scenario

\begin{tabular}{|l|c|c|c|}
\hline \multicolumn{4}{|c|}{ GSM network } \\
\hline Scenario & RANDOM & FAHP & COLLABORATIVE \\
\hline 2 channels & 12.62 & 50.19 & 84.52 \\
\hline 5 channels & 22.77 & 70.5 & 86.33 \\
\hline 10 channels & 32.02 & 71.06 & 91.22 \\
\hline 20 channels & 36.94 & 79.02 & 91.43 \\
\hline 30 channels & 34.43 & 78.78 & 90.85 \\
\hline 40 channels & 29.61 & 79 & 90.22 \\
\hline 50 channels & 24.6 & 81.6 & 83.02 \\
\hline Total Score & 31.25 & 82.6 & 100 \\
\hline
\end{tabular}

Source: Elaborated by the author

\section{CONCLUSIONS}

The developed algorithm presents an efficient performance in the metrics proposed for the cognitive distributed wireless networks, providing greater bandwidth for the cognitive users in high and low traffic. Moreover, it offers lower quantity of channel changes or number of handoff for the cognitive users in high traffic of primary users, regarding the selected algorithms (random and FAHP-RT algorithms) for the validation with real spectrum data of the developed Collaborative FAHP algorithm.

A simulator was designed for the allocation in cognitive distributed wireless networks that evaluates the algorithms, obtaining metrics based on real spectrum data of GSM frequency band that included spectrum ranges 100-1900 MHz, 1900-1950 MHz and 1959-2000 MHz, which depicts the work of each algorithm concerning the bandwidth that it offers to the cognitive users and how many handoffs it implements, taking into account the quantity of primary users presented in the network.

Finally, the dynamic access to the spectrum has received greater importance in the CR and as future results; they are expected not to depend on the artificial intelligence. Inasmuch as the greatest profit from the communication networks it will be able to bear a re-organized infrastructure that may manage the elements of the network continuously, ensuring the improvement of the system agents' service quality. However, the application of artificial intelligence techniques must be improved and extended in a meta-cognitive process.

\section{ACKNOWLEDGEMENTS}

This work is the results of a research project funded by Universidad ECCI through the internal call $\mathrm{N}^{\mathrm{o}} 1$ of 2014-2015.

\section{REFERENCES}

[1] S. Haykin, "Cognitive radio: Brain-empowered wireless communications," IEEE J. Sel. Areas Commun., vol. 23, no. 2, pp. 201-220, 2005.

[2] D. Xenakis, N. Passas, and L. Merakos, "Multi-parameter performance analysis for decentralized cognitive radio networks," Wirel. networks, vol. 20, no. 4, pp. 787-803, 2014. 
[3] M. Caleffi, I. F. Akyildiz, and L. Paura, "OPERA: Optimal routing metric for cognitive radio ad hoc networks," Wirel. Commun. IEEE Trans., vol. 11, no. 8, pp. 2884-2894, 2012.

[4] S. Abdelaziz and M. ElNainay, "Metric-based taxonomy of routing protocols for cognitive radio ad hoc networks," J. Netw. Comput. Appl., vol. 40, pp. 151-163, 2014.

[5] A. S. Cacciapuoti, L. P. Marcello, and M. A. Rahman, "Channel availability for mobile cognitive radio networks," J. Netw. Comput. Appl., vol. 47, pp. 131-136, 2015.

[6] M. Hashem, S. I. Barakat, and M. A. AttaAlla, "Distributed channel selection based on channel weight for cognitive radio network," in Computer Engineering Conference (ICENCO), 2014 10th International, 2014, pp. 115-120.

[7] M. Hasegawa, H. Hirai, K. Nagano, H. Harada, and K. Aihara, "Optimization for centralized and decentralized cognitive radio networks," Proc. IEEE, vol. 102, no. 4, pp. 574-584, 2014.

[8] Y. Liu, Z. Y. Feng, and P. Zhang, "Optimized in-band control channel with channel selection scheduling and network coding in distributed cognitive radio networks," J. China Univ. Posts Telecommun., vol. 19, no. 2, pp. 48-56, 2012.

[9] M. Naeem, A. Anpalagan, M. Jaseemuddin, and D. C. Lee, "Resource allocation techniques in cooperative cognitive radio networks," IEEE Commun. Surv. Tutorials, vol. 16, no. 2, pp. 729-744, 2014.

[10] N. Saranya, W. E. Santhkumar, and E. D. K. Ruby, "A survey on distributed channel selection technique using surf algorithm for information transfer in multi-hop cognitive radio networks," Proc. - 2014 Int. Conf. Comput. Sci. Comput. Intell. CSCI 2014, vol. 1, pp. 96-100, 2014.

[11] M. H. Rehmani, A. C. Viana, H. Khalife, and S. Fdida, "SURF: A distributed channel selection strategy for data dissemination in multi-hop cognitive radio networks," Comput. Commun., vol. 36, no. 10-11, pp. 1172-1185, 2013.

[12] S. Debroy, S. De, and M. Chatterjee, "Contention based multichannel MAC protocol for distributed cognitive radio networks," IEEE Trans. Mob. Comput., vol. 13, no. 12, pp. 2749-2762, 2014.

[13] L. F. Pedraza, A. Molina, and I. Paez, "Spectrum occupancy statistics in Bogota-Colombia," in IEEE Colombian Conference on Communications and Computing, 2013, pp. 1-6.

[14] G. Büyüközkan, C. Kahraman, and D. Ruan, "A fuzzy multi-criteria decision approach for software development strategy selection," Int. J. Gen. Syst., vol. 33, no. 2-3, pp. 259-280, 2004.

[15] E. Miranda, "Improving subjective estimates using paired comparisons," IEEE Softw., vol. 18, no. 1, pp. 87-91, 2001.

\section{AUTHOR PROFILE}

C. Salgado Electronic and Telecommunications Engineer, Master in Science in Information and Communication Sciences, Teacher and Researcher from Universidad ECCI, Colombia, Bogotá. Contact lsalgadof@ecci.edu.co

Sergio Mora Electronic Engineer, Master in Computer Sciences and Electronic. Teacher and Researcher from Universidad ECCI, Colombia, Bogotá. Contact: smoram@ecci.edu.co

Diego Giral Electronic Engineer, Master in Electronic Engineer, Teacher in Universidad Distrital Francisco Jose de Caldas, Bogotá, Colombia. Contact: diego.a.giral@gmail.com 